\title{
Random walks on generating sets for finite groups
}

\author{
F. R. K. Chung ${ }^{1}$ \\ University of Pennsylvania \\ Philadelphia, PA 19104 \\ R. L. Graham \\ AT\&T Research \\ Murray Hill, NJ 07974
}

Submitted: August 31, 1996; Accepted: November 12, 1996

Dedicated to Herb Wilf on the occasion of his sixty-fifth birthday

\begin{abstract}
We analyze a certain random walk on the cartesian product $G^{n}$ of a finite group $G$ which is often used for generating random elements from $G$. In particular, we show that the mixing time of the walk is at most $c_{r} n^{2} \log n$ where the constant $c_{r}$ depends only on the order $r$ of $G$.
\end{abstract}

\section{Introduction}

One method often used in computational group theory for generating random elements from a given (non-trivial) finite group $G$ proceeds as follows (e.g., see [2]]. A fixed integer $n \geq 2$ is initially specified. Denote by $G^{n}$ the set $\left\{\left(x_{1}, \ldots, x_{n}\right): x_{i} \in G, 1 \leq i \leq n\right\}$. If $\bar{x}=\left(x_{1}, \ldots, x_{n}\right) \in G^{n}$, we denote by $\langle\bar{x}\rangle$ the subgroup of $G$ generated by $\left\{x_{i}: 1 \leq i \leq n\right\}$. Let $G^{*} \subseteq G^{n}$ denote the set of all $\bar{x} \in G^{n}$ such that $\langle\bar{x}\rangle=G$. We execute a random walk on $G^{*}$ by taking the following general step. Suppose we are at a point $\bar{p}=\left(p_{1}, \ldots, p_{n}\right) \in G^{*}$. Choose a random pair of indices $(i, j)$ with $i \neq j$. (Thus, each such pair is chosen with probability $\frac{1}{n(n-1)}$.) We then move to one of $\bar{p}^{\prime}=\left(p_{1}^{\prime}, \ldots, p_{n}^{\prime}\right)$ where

$$
p_{k}^{\prime}= \begin{cases}p_{i} p_{j} \text { or } p_{i} p_{j}^{-1} & \text { if } k=i, \text { each with probability } 1 / 2 \\ p_{k} & \text { if } k \neq i .\end{cases}
$$

This rule determines the corresponding transition matrix $Q$ of the walk. We note that with this rule, we always have $\bar{p}^{\prime} \in G^{*}$. It is also easy to check that for $n \geq n_{0}(G)$, this walk is irreducible and aperiodic (see Section 5 for more quantitative remarks), and has a stationary distribution $\pi$ which is uniform (since $G^{*}$ is a multigraph in which every vertex has degree $2 n(n-1))$.

\footnotetext{
${ }^{1}$ Research supported in part by NSF Grant No. DMS 95-04834
} 
THE ELECTRONIC JOURNAL OF COMBINATORICS 4, NO. 2 (1997), \#R7

Starting from some fixed initial distribution $f_{0}$ on $G^{*}$, we apply this procedure some number of times, say $t$, to reach a distribution $f_{0} Q^{t}$ on $G^{*}$ which we hope will be close to "random" when $t$ is large. A crucial question which must be faced in this situation is just how rapidly this process mixes, i.e., how large must $t$ be so that $f_{0} Q^{t}$ is close to uniform. In this note, we apply several rather general comparison theorems to give reasonably good bounds on the mixing time for $Q$. In particular, we show (see Theorem 1) that when $t \geq c(G) n^{2} \log n$, where $c(G)$ is a constant depending only on $G$, then $Q^{t}$ is already quite close to uniform (where we usually will suppress $f_{0}$ ).

This problem belongs to a general class of random walk problems suggested recently by David Aldous [1]. In fact, he considers a more general walk in which only certain pairs of indices $(i, j)$ are allowed in forming $p_{k}^{\prime}=p_{i} p_{j}$ or $p_{i} p_{j}^{-1}$. These pairs can be described by a graph $H$ on the vertex set $\{1,2, \cdot, n\}$. The case studied in this note corresponds to taking $H$ to be a complete graph.

We first learned of this problem from a preprint of Diaconis and Saloff-Coste [6], part of which has subsequently appeared [7]. In it, they wrote " $\cdots$ for $G=\mathbb{Z}_{p}$ with $p=2,3,4,5,7,8,9$ we know that $n^{2} \log n$ steps are enough whereas for $G=\mathbb{Z}_{6}$ or $\mathbb{Z}_{10}$ we only know that $n^{4} \log n$ are enough. Even in the case of $\mathbb{Z}_{6}$ it does not seem easy to improve this." Our main contribution in this note is to show that by direct combinatorial constructions, a mixing time of $c(G) n^{2} \log n$ can be obtained for all groups $G$ where $c(G)$ is a constant depending just on $G$. Subsequently, they have now [8] also obtained bounds of the form $c(G) n^{2} \log n$ for all groups $G$ by including a more sophisticated path construction argument than they had previously used in [6].

\section{Background}

A weighted graph $\Gamma=(V, E)$ consists of a vertex set $V$, and a weight function $w: V \times V \rightarrow \mathbb{R}$ satisfying $w(u, v)=w(v, u) \geq 0$ for all $u, v \in V$. The edge set $E$ of $\Gamma$ is defined to be the set of all pairs $u v$ with $w(u, v)>0$. A simple (unweighted) graph is just the special case in which all weights are 0 or 1 . The degree $d_{v}$ of a vertex $v$ is defined by

$$
d_{v}:=\sum_{u} w(u, v)
$$


Further, we define the $|V| \times|V|$ matrix $L$ by

$$
L(u, v)=\left\{\begin{array}{cl}
d_{v}-w(v, v) & \text { if } u=v \\
-w(u, v) & \text { if } u v \in E, u \neq v \\
0 & \text { otherwise }
\end{array}\right.
$$

In particular, for a function $f: V \rightarrow \mathbb{R}$, we have

$$
L f(x)=\sum_{\substack{y \\ x y \in E}}(f(x)-f(y)) w(x, y) .
$$

Let $T$ denote the diagonal matrix with the $(v, v)$ entry having the value $d_{v}$. The Laplacian $\mathcal{L}_{\Gamma}$ of $\Gamma$ is defined to be

$$
\mathcal{L}=\mathcal{L}_{\Gamma}=T^{-1 / 2} L T^{-1 / 2}
$$

In other words,

$$
\mathcal{L}(u, v)=\left\{\begin{array}{cl}
1-\frac{w(v, v)}{d_{v}} & \text { if } u=v \\
-\frac{w(u, v)}{\sqrt{d_{u} d_{v}}} & \text { if } u v \in E, u \neq v \\
0 & \text { otherwise }
\end{array}\right.
$$

Since $\mathcal{L}$ is symmetric and non-negative definite, its eigenvalues are real and non-negative. We denote them by

$$
0=\lambda_{0} \leq \lambda_{1} \leq \cdots \leq \lambda_{n-1}
$$

where $n=|V|$.

It follows from standard variational characterizations of eigenvalues that

$$
\lambda_{1}=\inf _{f} \sup _{c} \frac{\sum_{u, v \in E}(f(u)-f(v))^{2} w(u, v)}{\sum_{x} d_{x}(f(x)-c)^{2}} .
$$

For a connected graph $\Gamma$, the eigenvalues satisfy

$$
0<\lambda_{i} \leq 2
$$

for $i \geq 1$. Various properties of the eigenvalues can be found in [3].

Now, the usual random walk on an unweighted graph has transition probability $1 / d_{v}$ of moving from a vertex $v$ to any one of its neighbors. The transition matrix $P$ then satisfies

$$
P(v, u)=\left\{\begin{array}{cl}
1 / d_{v} & \text { if } u v \in E \\
0 & \text { otherwise }
\end{array}\right.
$$

That is,

$$
f P(u)=\sum_{\substack{v \\ u v \in E}} \frac{1}{d_{v}} f(v)
$$


THE ELECTRONIC JOURNAL OF COMBINATORICS 4, NO. 2 (1997), \#R7

for any $f: V \rightarrow \mathbb{R}$. It is easy to check that

$$
P=T^{-1 / 2}(I-R) T^{1 / 2}=T^{-1} A
$$

where $A$ is the adjacency matrix of the graph.

In a random walk on a connected weighted graph $\Gamma$, the transition matrix $P$ satisfies

$$
1 T P=1 T
$$

Thus, the stationary distribution is just $1 T / \operatorname{vol}(\Gamma)$, where $\operatorname{vol}(\Gamma)=\sum_{x} d_{x}$ and $\mathbf{1}$ is the all ones vector. Our problem is to estimate how rapidly $f P^{k}$ converges to its stationary distribution, as $k \rightarrow \infty$, starting from some initial distribution $f: V \rightarrow \mathbb{R}$. First, consider convergence in the $L^{2}$ (or Euclidean) norm. Suppose we write

$$
f T^{-1 / 2}=\sum_{i} a_{i} \phi_{i}
$$

where $\phi_{i}$ denotes the eigenfunction associated with $\lambda_{i}$ and $\left\|\phi_{i}\right\|=1$. Since $\phi_{0}=1 \cdot T^{1 / 2} / \sqrt{\operatorname{vol}(\Gamma)}$ then

$$
a_{0}=\frac{\left\langle f T^{-1 / 2}, 1 T^{1 / 2}\right\rangle}{\left\|1 T^{1 / 2}\right\|}=\frac{1}{\sqrt{\operatorname{vol}(\Gamma)}}
$$

since $\langle f, 1\rangle=1$. We then have

$$
\begin{aligned}
\left\|f P^{s}-1 T / \operatorname{vol}(\Gamma)\right\| & =\left\|f T^{-1 / 2}(I-\mathcal{L})^{s} T^{1 / 2}-a_{0} \phi_{0} T^{1 / 2}\right\| \\
& =\left\|\sum_{i \neq 0}\left(1-\lambda_{i}\right)^{s} a_{i} \phi_{i} T^{1 / 2}\right\| \\
& \leq(1-\lambda)^{s}\|f\| \\
& \leq e^{-s \lambda}\|f\|
\end{aligned}
$$

where

$$
\lambda=\left\{\begin{array}{cl}
\lambda_{1} & \text { if } 1-\lambda_{1} \geq \lambda_{n-1}-1, \\
2-\lambda_{n-1} & \text { otherwise } .
\end{array}\right.
$$

So, after $s \geq(1 / \lambda) \log (1 / \epsilon)$ steps, the $L_{2}$ distance between $f P^{s}$ and its stationary distribution is at most $\epsilon\|f\|$.

Although $\lambda$ occurs in the above bound, in fact only $\lambda_{1}$ is crucial, in the following sense. If it happens that $1-\lambda_{1}<\lambda_{n-1}-1$, then we can consider a random walk on the modified graph $\Gamma^{\prime}$ formed by adding a loop of weight $c d_{v}$ to each vertex $v$ where $c=\left(\lambda_{1}+\lambda_{n-1}\right) / 2-1$. The new graph has (Laplacian) eigenvalues $\lambda_{k}^{\prime}=\frac{1}{1+c} \lambda_{k} \leq 1,0 \leq k \leq n-1$, so that $1-\lambda_{1}^{\prime} \geq \lambda_{n-1}^{\prime}-1$. 
Consequently (see [3]), we only need to increase the number of steps of this "lazy" walk on $\Gamma$ to $s \geq\left(1 /\left(\lambda^{\prime}\right) \log (1 / \epsilon)\right.$ to achieve that same $L_{2}$ bound on $\epsilon\|f\|$ where $\lambda^{\prime}$ is

$$
\lambda^{\prime}= \begin{cases}\lambda_{1} & \text { if } 1-\lambda_{1} \geq \lambda_{n-1}-1, \\ \frac{2 \lambda_{1}}{\lambda_{1}+\lambda_{n-1}} & \text { otherwise }\end{cases}
$$

We note that we have $\lambda^{\prime} \geq 2 \lambda_{1} /\left(2+\lambda_{1}\right) \geq 2 \lambda_{1} / 3$.

A stronger notion of convergence is measured by the $L_{\infty}$, or relative pointwise distance, which is defined as follows. After $s$ steps, the relative pointwise distance of $P$ to its stationary distribution $\pi$ is given by

$$
\Delta(s):=\max _{x, y} \frac{\left|P^{s}(y, x)-\pi(x)\right|}{\pi(x)} .
$$

Let $\delta_{z}$ denote the indicator function defined by

$$
\delta_{z}(x)= \begin{cases}1 & \text { if } x=z \\ 0 & \text { otherwise }\end{cases}
$$

Set

$$
T^{1 / 2} \delta_{x}=\sum_{i} a_{i} \phi_{i}
$$

and

$$
T^{-1 / 2} \delta_{y}=\sum_{i} \beta_{i} \phi_{i}
$$

In particular,

$$
\alpha_{0}=\frac{d_{x}}{\sqrt{\operatorname{vol}(\Gamma)}}, \quad \beta_{0}=\frac{1}{\sqrt{\operatorname{vol}(\Gamma)}} .
$$

Hence,

$$
\begin{aligned}
\Delta(t) & =\max _{x, y} \frac{\left|\delta_{y}\left(P^{t}\right) \delta_{y}-\pi(x)\right|}{\pi(x)} \\
& =\max _{x, y} \frac{\left|\delta_{y} T^{-1 / 2}(I-\mathcal{L})^{t} T^{1 / 2} \delta_{x}-\pi(x)\right|}{\pi(x)} \\
& \leq \max _{x, y} \sum_{i \neq 0} \frac{\left|\left(1-\lambda_{i}\right)^{t} \alpha_{i} \beta_{i}\right|}{d_{x} / \operatorname{vol}(\Gamma)} \\
& \leq(1-\lambda)^{t} \max _{x, y} \frac{\left\|T^{1 / 2} \delta_{x}\right\|\left\|T^{-1 / 2} \delta_{y}\right\|}{d_{x} / \operatorname{vol}(\Gamma)} \\
& \leq(1-\lambda)^{t} \frac{\operatorname{vol}(\Gamma)}{\min _{x, y} \sqrt{d_{x} d_{y}}} \\
& \leq e^{-t \lambda} \frac{\operatorname{vol}(\Gamma)}{\min _{x} d_{x}} .
\end{aligned}
$$

Thus, if we choose $t$ so that

$$
t \geq \frac{1}{\lambda} \log \frac{\operatorname{vol}(\Gamma)}{e \min _{x} d_{x}}
$$


THE ELECTRONIC JOURNAL OF COMBINATORICS 4, NO. 2 (1997), \#R7

then after $t$ steps, we have $\Delta(t) \leq \epsilon$. We also remark that requiring $\Delta(t) \rightarrow 0$ is a rather strong condition. In particular, it implies that another common measure, the total variation distance $\Delta_{T V}(t)$ goes to zero just as rapidly, since

$$
\begin{aligned}
\Delta_{T V}(t) & =\max _{A \subset V} \max _{y \in V}\left|\sum_{x \in A} P^{t}(y, x)-\pi(x)\right| \\
& \leq \max _{\substack{A \subset V \\
v o l(A) \leq \frac{1}{2} \operatorname{vol}(\Gamma)}} \sum_{x \in A} \pi(x) \Delta(t) \\
& \leq \frac{1}{2} \Delta(t) .
\end{aligned}
$$

We point out here that the factor $\frac{\operatorname{vol}(\Gamma)}{\min _{x} d_{x}}$ can often be further reduced by the use of so-called logarithmic Sobolev eigenvalue bounds (see [9] and [3] for surveys). In particular, Diaconis and Saloffe-Coste have used these methods in their work on rapidly mixing Markov chains. We will follow their lead and apply some of these ideas in Section 4.

\section{An eigenvalue comparison theorem}

To estimate the rate at which $\Delta(t) \rightarrow 0$ as $t \rightarrow \infty$, we will need to lower bound $\lambda_{1}\left(\Gamma^{*}\right)$, the smallest non-zero Laplacian eigenvalue of the graph $\Gamma^{*}$ on $G^{*}$, defined by taking as edges all pairs $\bar{x} \bar{y} \in E^{*}$ where $\bar{x} \in G^{*}$ and $\bar{y}$ can be reached from $\bar{x}$ by taking one step of the process $Q$. Our comparison graph $\Gamma^{n}$ on $G^{n}$ will have all edges $\bar{x} \bar{y} \in E$ where $\bar{x}$ and $\bar{y}$ are any two elements of $G^{n}$ which differ in a single coordinate (so that $\Gamma^{n}$ is just the usual Cartesian product of $G$ with itself $n$ times).

Lemma 1. Suppose $\Gamma=(V, E)$ is a connected (simple) graph and $\Gamma^{\prime}=\left(V^{\prime}, E^{\prime}\right)$ is a connected multigraph with Laplacian eigenvalues $\lambda_{1}=\lambda_{1}(\Gamma)$ and $\lambda_{1}^{\prime}=\lambda_{1}\left(\Gamma^{\prime}\right)$, respectively. Suppose $\phi: V \rightarrow V^{\prime}$ is a surjective map such that:

(i) If $d_{x}$ and $d_{x^{\prime}}^{\prime}$ denote the degrees of $v \in V$ and $x^{\prime} \in V^{\prime}$, respectively, then for all $x^{\prime} \in V^{\prime}$ we have

$$
\sum_{x \in \phi^{-1}\left(x^{\prime}\right)} d_{x} \geq a d_{x^{\prime}}^{\prime}
$$

(ii) For each edge $e=x y \in E$ there is a path $P(e)$ between $\phi(x)$ and $\phi(y)$ in $E^{\prime}$ such that:

(a) The number of edges of $P(e)$ is at most $\ell$;

(b) For each edge $e^{\prime} \in E^{\prime}$, we have

$$
\mid\left\{x y \in E: e^{\prime} \in P(e) \mid \leq m\right.
$$


THE ELECTRONIC JOURNAL OF COMBINATORICS 4, NO. 2 (1997), \#R7

Then we have

$$
\lambda_{1}^{\prime} \geq \frac{a}{\ell m} \lambda_{1}
$$

Proof. For $h: V \rightarrow \mathbb{C}$, define $h^{2}: E \rightarrow \mathbb{C}$ by setting $h^{2}(e)=(h(x)-h(y))^{2}$ for $e=x y \in E$ (with a similar definition for $h: V^{\prime} \rightarrow \mathbb{C}$ and $h^{2}: E^{\prime} \rightarrow \mathbb{C}$ ).

We start by letting $g: V^{\prime} \rightarrow \mathbb{C}$ be a function achieving equality in (1) or rather, the version of (1) for $\left.\lambda_{1}^{\prime}\right)$. Define $f: V \rightarrow \mathbb{C}$ by setting

$$
f(x)=g(\phi(x)) \text { for } \quad x \in V .
$$

Thus,

$$
\begin{aligned}
\lambda_{1}^{\prime} & =\sup _{c} \frac{\sum_{e^{\prime} \in E^{\prime}} g^{2}\left(e^{\prime}\right)}{\sum_{v^{\prime} \in V^{\prime}}\left(g\left(v^{\prime}\right)-c\right)^{2} d_{v^{\prime}}^{\prime}} \\
& \geq \frac{\sum_{e^{\prime} \in E^{\prime}} g^{2}\left(e^{\prime}\right)}{\sum_{v^{\prime} \in V^{\prime}}\left(g\left(v^{\prime}\right)-c\right)^{2} d_{v^{\prime}}^{\prime}} \text { for all } c \\
& =\frac{\sum_{e^{\prime} \in E^{\prime}} g^{2}\left(e^{\prime}\right)}{\sum_{e \in E} f^{2}(e)} \cdot \frac{\sum_{e \in E} f^{2}(e)}{\sum_{v \in V}(f(v)-c)^{2} d_{v}} \cdot \frac{\sum_{v \in V}(f(v)-c)^{2} d_{v}}{\sum_{v^{\prime} \in V^{\prime}}\left(g\left(v^{\prime}\right)-c\right)^{2} d_{v^{\prime}}^{\prime}} \\
& =I \times I I \times I I I .
\end{aligned}
$$

First, we treat factor $I$. Using Cauchy-Schwarz, we have for all $e \in E$,

$$
f^{2}(e) \leq \ell \sum_{e^{\prime} \in P(e)} g^{2}\left(e^{\prime}\right)
$$

by (a). Hence by (b).

$$
m \sum_{e^{\prime} \in E} g^{2}\left(e^{\prime}\right) \geq \sum_{e \in E} \sum_{e^{\prime} \in E^{\prime}} g^{2}\left(e^{\prime}\right) \geq \frac{1}{\ell} \sum_{e \in E} f^{2}(e)
$$

i.e.,

$$
\frac{\sum_{e^{\prime} \in E^{\prime}} g^{2}\left(e^{\prime}\right)}{\sum_{e \in E} f^{2}(e)} \geq \frac{1}{\ell m}
$$

which gives a bound for factor $I$. To bound factor $I I I$, we have

$$
\begin{aligned}
\sum_{x \in V}(f(x)-c)^{2} d_{x} & =\sum_{x^{\prime} \in V^{\prime}} \sum_{x \in \phi^{-1}\left(x^{\prime}\right)}(f(x)-c)^{2} d_{x} \\
& =\sum_{x^{\prime} \in V^{\prime}}\left(g\left(x^{\prime}\right)-c\right)^{2} \sum_{x \in \phi^{-1}\left(x^{\prime}\right)} d_{x} \\
& \geq a \sum_{x^{\prime} \in V^{\prime}}\left(g\left(x^{\prime}\right)-c\right)^{2} d_{x^{\prime}}^{\prime} \quad \text { by }(\mathrm{i}) .
\end{aligned}
$$


THE ELECTRONIC JOURNAL OF COMBINATORICS 4, NO. 2 (1997), \#R7

Finally, for factor $I I$ we choose $c_{0}$ so that

$$
\sup _{c} \frac{\sum_{e \in E} f^{2}(e)}{\sum_{v \in V}(f(v)-c)^{2} d_{v}}=\frac{\sum_{e \in E} f^{2}(e)}{\sum_{v \in V}\left(f(v)-c_{0}\right)^{2} d_{v}} \geq \lambda_{1}
$$

by $(1)$

Hence, by (4) (5) and (7) we have

$$
\lambda_{1}^{\prime} \geq \frac{a}{\ell m} \lambda_{1}
$$

which is just (3).

Note that in the case that $\Gamma$ and $\Gamma^{\prime}$ are regular with degrees $k$ and $k^{\prime}$, respectively, then (i) holds with $a=k / k^{\prime}$, and $(3)$ becomes

$$
\lambda_{1}^{\prime} \geq \frac{k}{k^{\prime} \ell m} \lambda_{1}
$$

\section{A comparison theorem for the log-Sobolev constant}

Given a connected weighted graph $\Gamma=(V, E)$, the log-Sobolev constant $\alpha=\alpha(\Gamma)$ is defined by

$$
\alpha=\inf _{f \neq \text { constant }} \frac{\sum_{e \in E} f^{2}(e)}{\sum_{x} f^{2}(x) d_{x} \log \frac{f^{2}(x)}{\sum_{y} f^{2}(y) \pi(y)}}
$$

where $f$ ranges over all non-constant functions $f: V \rightarrow \mathbb{R}$ and $\pi$ is the stationary distribution of the nearest neighbor random walk on $\Gamma$. In a recent paper [9], Diaconis and Saloffe-Coste show that

$$
\Delta_{T V}(t) \leq e^{1-c} \quad \text { if } \quad t \geq \frac{1}{2 \alpha} \log \log \frac{\operatorname{vol}(\Gamma)}{\min _{x} d_{x}}+\frac{c}{\lambda_{1}} .
$$

This is strengthened in [3], where the slightly stronger inequality is proved

$$
\Delta(t) \leq e^{2-c} \quad \text { if } t \geq \frac{1}{2 \alpha} \log \log \frac{\operatorname{vol}(\Gamma)}{\min _{x} d_{x}}+\frac{c}{\lambda_{1}}
$$

and

$$
\Delta_{T V}(t) \leq e^{1-c} \text { if } t \geq \frac{1}{4 \alpha} \log \log \frac{\operatorname{vol}(\Gamma)}{\min _{x} d_{x}}+\frac{c}{\lambda_{1}}
$$

using the alternate (equivalent) definition:

$$
\alpha=\inf _{f \neq \text { constant }} \frac{\sum_{e \in E} f^{2}(e)}{S(f)}
$$


THE ELECTRONIC JOURNAL OF COMBINATORICS 4, NO. 2 (1997), \#R7

where

$$
S(f):=\inf _{c>0} \sum_{x \in V}\left(f^{2}(x) \log f^{2}(x)-f^{2}(x)-f^{2}(x) \log c+c\right) d_{x} .
$$

While (10) is typically stronger than (2), it depends on knowing (or estimating) the value of $\alpha$, which if anything is harder to estimate than $\lambda_{1}$ for general graphs. We can bypass this difficulty to some extent by the following (companion) comparison theorem for $\alpha$. Its statement (and proof) is in fact quite close to that of Lemma 1.

Lemma 2. Suppose $\Gamma=(V, E)$ is a connected (simple) graph and $\Gamma^{\prime}=\left(V^{\prime}, E^{\prime}\right)$ is a connected multigraph, with logarithmic Sobolev constants $\alpha=\alpha(\Gamma)$ and $\alpha^{\prime}=\alpha\left(\Gamma^{\prime}\right)$, respectively. Suppose $\phi: V \rightarrow V^{\prime}$ is a surjective map such that (i), (ii) and (iii) of Lemma 1 hold. Then

$$
\alpha^{\prime} \geq \frac{a}{\ell m} \alpha
$$

Proof: Consider a function $g: V^{\prime} \rightarrow \mathbb{R}$ achieving equality in (14). Define $f: V \rightarrow \mathbb{R}$ as in the proof of Lemma 1. Then we have

$$
\begin{aligned}
\alpha^{\prime} & =\frac{\sum_{e^{\prime} \in E^{\prime}} g^{2}\left(e^{\prime}\right)}{S(g)} \\
& =\frac{\sum_{e^{\prime} \in E^{\prime}} g^{2}\left(e^{\prime}\right)}{\sum_{e \in E} f^{2}(e)} \cdot \frac{\sum_{e \in E} f^{2}(e)}{S(f)} \cdot \frac{S(f)}{S(g)} \\
& =I^{\prime} \times I I^{\prime} \times I I I^{\prime}
\end{aligned}
$$

Exactly as in the proof of Lemma 1, we obtain

$$
I^{\prime} \geq \frac{1}{\ell m}, \quad I I^{\prime} \geq \alpha
$$

It remains to show $I I I^{\prime} \geq a$ (which we do using a nice idea of Holley and Stroock; cf. [9]). First, define

$$
F(\xi, \zeta):=\xi \log \xi-\xi \log \zeta-\xi+\zeta
$$

for all $\xi, \zeta>0$. Note that $F(\xi, \zeta) \geq 0$ and for $\zeta>0, F(\xi, \zeta)$ is convex in $\xi$. Thus, for some $c_{0}>0$,

$$
\begin{aligned}
S(f) & =\sum_{x \in V} F\left(f^{2}(x), c_{0}\right) d_{x} \\
& =\sum_{x^{\prime} \in V^{\prime}}\left(\sum_{x \in \phi^{-1}\left(x^{\prime}\right)} d_{x}\right) F\left(g\left(x^{\prime}\right)^{2}\right)
\end{aligned}
$$


THE ELECTRONiC JOURNAL OF COMBINATORICS 4, NO. 2 (1997), \#R7

$$
\begin{aligned}
& \geq \sum_{x^{\prime} \in V^{\prime}} a d_{x^{\prime}}^{\prime} F\left(g\left(x^{\prime}\right)^{2}\right) \quad \text { since } F \geq 0 \\
& \geq a \sum_{x^{\prime} \in V^{\prime}} F\left(g\left(x^{\prime}\right)^{2} d_{x^{\prime}}^{\prime}\right) \quad \text { by convexity } \\
& =a S(g) .
\end{aligned}
$$

This implies $I I I^{\prime} \geq a$ and (14) is proved.

As in $\left(3^{\prime}\right)$ if $\Gamma$ and $\Gamma^{\prime}$ are regular with degrees $k$ and $k^{\prime}$, respectively, then

$$
\alpha^{\prime} \geq \frac{k}{k^{\prime} \ell m} \alpha
$$

\section{Defining the paths}

In this section we describe the key path constructions for our proof. For our finite group $G$, we say that $B \subseteq G$ is a minimal basis for $G$ if $\langle B\rangle=G$ but for any proper subset $B^{\prime} \subset B$, we have $\left\langle B^{\prime}\right\rangle \neq G$. Define

$$
b(G):=\max \{|B|: B \text { is a minimal basis for } G\} .
$$

Further, define $w(G)$ to be the least integer such that for any minimal basis $B$, and any $g \in G$, we can write $g$ as a product of at most $w$ terms of the form $x^{ \pm 1}, x \in B$. Finally, define $s(G)$ to be the cardinality of a minimum basis for $G$. We abbreviate $b(G), w(G)$ and $s(G)$ by $b, w$ and $s$, respectively, and, as usual, we set $r:=|G|$. In particular, the following crude bounds always hold:

$$
s \leq b \leq \frac{\log r}{\log 2}=\log _{2} r, \quad w<r .
$$

Let $R$ denote $\left\lfloor\log _{2} r\right\rfloor$. We will assume $n>2(s+R)$. To apply Lemmas 1 and 2 , we must define the map $\phi: \Gamma^{n} \rightarrow \Gamma^{*}$ and the paths $P(e), e \in E^{n}$. Let $\left\{g_{1}, \ldots, g_{s}\right\}$ be a fixed minimum basis for $G$.

For $\bar{x}=\left(x_{1}, \ldots, x_{n}\right) \in \Gamma^{n}$, define

$$
\phi(\bar{x})=\left\{\begin{array}{cl}
\bar{x} & \text { if }\langle\bar{x}\rangle=G, \\
\left(g_{1}, \ldots, g_{s}, x_{s+1}, \ldots, x_{n}\right) & \text { if }\langle\bar{x}\rangle \neq G .
\end{array}\right.
$$

Next, for each edge $e=\bar{x} \bar{y} \in E^{n}$, we must define a path $P(e)$ between $\phi(\bar{x})$ and $\phi(\bar{y})$ in $\Gamma^{*}$. Suppose $\bar{x}$ and $\bar{y}$ just differ in the $i^{\text {th }}$ component so that

$$
\bar{x}=\left(x_{1}, \ldots, x_{i}, \ldots, x_{n}\right), \quad \bar{y}=\left(y_{1}, \ldots, y_{i}, \ldots, y_{n}\right)
$$

where $x_{j}=y_{j}$ for $j \neq i$, and $x_{i} \neq y_{i}$. There are three cases: 
THE ELECTRONIC JOURNAL OF COMBINATORICS 4, NO. 2 (1997), \#R7

(I) $\overline{\boldsymbol{x}} \in \boldsymbol{G}^{*}, \overline{\boldsymbol{y}} \in \boldsymbol{G}^{*}$. Let $I$ denote interval

$$
\begin{cases}\{i+1, \ldots, i+s+2 R\} & \text { if } i \leq n-s-2 R, \\ \{n-s-2 R, \ldots, n\} \backslash\{i\} & \text { if } i>n-s-2 R .\end{cases}
$$

Choose a subset $J \subset I$ so that:

$$
\begin{aligned}
\text { (i) } & |J|=s \\
(\text { ii }) & \left\langle\left\{x_{k}: k \in[n] \backslash|J|\right\}\right\rangle=G \\
\text { (iii) } & \left\langle\left\{y_{k}: k \in[n] \backslash|J|\right\}\right\rangle=G .
\end{aligned}
$$

That is, the values $x_{j}=y_{j}, j \in J$, are not needed in generating $G$ using the $x_{k}$ or the $y_{k}$. Write $J$ as $\left\{j_{1}, j_{2}, \ldots, j_{s}\right\}$. In this case $\phi(\bar{x})=\bar{x}, \phi(\bar{y})=\bar{y}$. To form $P(e)$ :

(i) Use a basis from the elements $x_{k}, k \notin J$, to change $x_{j_{1}}$ to $g_{1}, x_{j_{2}}$ to $g_{2}, \ldots, x_{j_{s}}$ to $g_{s}$. This takes at most $w s$ steps;

(ii) Next, use $g_{1}, \ldots, g_{s}$ to change $x_{i}$ to $y_{i}$. This takes at most $w$ steps;

(iii) Finally, use a basis from the elements $y_{k}, k \notin J$, to change $g_{1}$ back to $x_{j_{1}}=y_{j_{1}}, \ldots, g_{s}$ back to $x_{j_{s}}=y_{j_{s}}$. This takes at most $w s$ steps. Hence, for case (I), $P(e)$ has length at most $w(2 s+1)$.

(II) $\overline{\boldsymbol{x}} \notin \boldsymbol{G}^{*}, \overline{\boldsymbol{y}} \in \boldsymbol{G}^{*}$. In this case, $\phi(\bar{x})=\left(g_{1}, \ldots, g_{s}, x_{s+1}, \ldots, x_{n}\right), \phi(\bar{y})=\left(y_{1}, \ldots, y_{n}\right)$ where $x_{j}=y_{j}$ for $j \neq i$, and $x_{i} \neq y_{i}$. This time we locate a set $J$ of $s$ indices $j_{1}, \ldots, j_{s}$, with $i<j_{1}<\cdots<j_{s} \leq i+s+R$ so that $\left\langle\left\{y_{k}: k \in[n] \backslash J\right\}\right\rangle=G$. If there is not enough room, i.e., $i>n-s-R$, then we locate $J$ to lie in $\{n-s-R, \ldots, n\} \backslash\{i\}$. In addition, if it happens that $i \leq s$, then we take $J \subseteq\{s+1, \ldots, 2 s+R\}$. Now, to form $P(e)$ :

(i) Use $g_{1}, \ldots, g_{s}$ in $\phi(\bar{x})$ to change $x_{j_{1}}$ to $g_{1}, x_{j_{2}}$ to $g_{2}, \ldots, x_{j_{s}}$ to $g_{s}$.

(ii) Use the newly formed $g_{1}, \ldots, g_{s}$ (with indices in $J$ ) to change coordinate 1 from $g_{1}$ to $y_{1}$, coordinate 2 from $g_{2}$ to $y_{2}, \ldots$, coordinate $s$ from $g_{s}$ to $y_{s}$. Then change $x_{i}$ to $y_{i}$.

(iii) Finally use a basis in $\left\{y_{k}: k \notin[n] \backslash J\right\}$ to change coordinates $j_{1}, \ldots, j_{s}$ to $y_{j_{1}}, \ldots, y_{j_{s}}$, respectively. In this case, the length of $P(e)$ is at most $w(3 s+1)$. 
THE ELECTRONIC JOURNAL OF COMBINATORICS 4, NO. 2 (1997), \#R7

III) $\overline{\boldsymbol{x}} \notin \boldsymbol{G}^{*}, \overline{\boldsymbol{y}} \notin \boldsymbol{G}^{*}$. Thus, $\phi(\bar{x})=\left(g_{1}, \ldots, g_{s}, x_{s+1}, \ldots, x_{n}\right), \phi(\bar{y})=\left(g_{1}, \ldots, g_{s}, y_{s+1}, \ldots, y_{n}\right)$ and of course, $x_{j}=y_{j}, j \neq i$, and $x_{i} \neq y_{i}$. If $i>s$ then we can change $x_{i}$ to $y_{i}$ in at most $w$ steps (and this forms $P(e)$ ). If $i \leq s$ then $\phi(\bar{x})=\phi(\bar{y})$ and $P(e)$ does not have to be defined.

The main point in the preceding slightly complicated construction is that it guarantees a rather small value of $m$. The reason is that the only coordinates $u$ which change in edges of $P(e)$ are either in $\{1,2, \ldots, s\}$ or fairly close to $i$, e.g. $|i-u| \leq 2(s+R)$. Furthermore, if a changing coordinate $u \in\{1, \ldots, s\}$ and $i>2(s+R)$, so that we have some edge $e^{\prime}=$ $\left(z_{1}, \ldots, z_{s}, \ldots, z_{u}, \ldots, z_{n}\right),\left(z_{1}, \ldots, z_{s}, \ldots, z_{u}^{\prime}, \ldots, z_{n}\right)$ in $\Gamma^{*}$, then we search $\left(z_{1}, \ldots, z_{n}\right)$ for the first interval of length $2(s+R)$ which contains $g_{1}, \ldots, g_{s}$, say $\{w+1, \ldots, w+2(s+R)\}$. By our construction, such an interval must exist.

Furthermore, it is not hard to see that in this case $|i-w|<4(s+R)$ (and this is somewhat generous). Consequently, the original point $\bar{x}$ in $e$ must agree with $\left(z_{1}, \ldots, z_{n}\right)$ in all but at most $10(s+R)$ coordinates. If follows that for these choices of $\phi$ and $P(e), e \in E^{n}$, we have

$$
m \leq 10(s+R) r^{10(s+R)}
$$

Also by previous remarks, we have

$$
\ell \leq w(3 s+1)
$$

Observe that $\operatorname{deg} \Gamma^{n}=(r-1) n$ and $\operatorname{deg} \Gamma^{*}=2 n(n-1)$.

Consequently, by $\left(3^{\prime}\right)$, $\left.13^{\prime}\right),(17)$ and (18) (after some simplifications)

$$
\lambda_{1}^{\prime} \geq \frac{\lambda_{1}}{1600 R^{2} r^{20 R} n}, \quad \alpha^{\prime} \geq \frac{\alpha}{1600 R^{2} r^{20 R} n} .
$$

\section{Putting it all together}

The final pieces we need to bound $\Delta(t)$ in (10) are the values of $\lambda_{1}\left(\Gamma^{n}\right)$ and $\alpha\left(\Gamma^{n}\right)$. Fortunately, these are easy to derive since $\lambda_{1}$ and $\alpha$ behave very nicely under Cartesian products. In particular, we have

$$
\lambda_{1}\left(\Gamma^{n}\right)=\alpha\left(\Gamma^{n}\right)=\frac{(r-1)}{r} \cdot \frac{1}{n} .
$$

Note that

$$
\operatorname{vol}\left(\Gamma^{*}\right) \leq \operatorname{vol}\left(\Gamma^{n}\right)=\left|\Gamma^{n}\right| \cdot 2 n(n-1) .
$$

Thus, by (10) we have

$$
\Delta(t) \leq e^{2-c} \quad \text { if } \quad t \geq \frac{1}{2 \alpha^{\prime}} \log \log \left|\Gamma^{n}\right|+\frac{c}{\lambda_{1}^{\prime}}
$$


and, by (11) we have

$$
\Delta_{T V}(t) \leq e^{1-c} \quad \text { if } \quad t \geq \frac{1}{4 \alpha^{\prime}} \log \log \left|\Gamma^{n}\right|+\frac{c}{\lambda_{1}^{\prime}}
$$

This implies

\section{Theorem 1.}

$$
\Delta(t) \leq e^{2-c} \quad \text { if } \quad t \geq 800 R^{2} r^{20 R+1} n^{2}(\log n+\log \log r+c)
$$

In other words, $c_{r} n^{2} \log n$ steps are enough to force the distribution to be close to uniform, which is what we claimed in the Introduction. Also, we have

\section{Theorem 2.}

$$
\Delta_{T V}(t) \leq e^{1-c} \quad \text { if } \quad t \geq 400 R^{2} r^{20 R+1} n^{2}(\log n+\log \log r+c)
$$

\section{Concluding remarks}

Of course, the preceding techniques using comparison theorems can be applied to many other random walk problems of this general type. For example, one could restrict the preceding moves so that $p_{i} \rightarrow p_{i} p_{j}^{ \pm 1}$ is only allowed if $(i, j)$ belongs to some specified set (this determines an underlying digraph).

It is probably true that the correct answer in (21) is actually $c_{r} n \log n$ (this is conjectured

in [6] . Some evidence in favor of this is our recent result in [4] that $O(n \log n)$ steps do suffice when $G=\mathbb{Z}_{2}$.

\section{References}

[1] David Aldous, talk at 1994 National IMS meeting.

[2] F. Celler, C. R. Leedham-Green, S. Murray, A. Niemeyer and E. A. Obrien, Generating random elements of a finite group, Comm. Alg., 23 (1995), 4931-4948.

[3] F. R. K. Chung, Spectral Graph Theory, Amer. Math. Soc., Providence, RI (to appear 1996).

[4] F. R. K. Chung and R. L. Graham, Stratified random walk on an $n$-cube, preprint. 
THE ELECTRONIC JOURNAL OF COMBINATORICS 4, NO. 2 (1997), \#R7

[5] P. Diaconis, R. L. Graham and J. A. Morrison, Asymptotic analysis of a random walk on a hypercube with many dimensions, Random Structures and Algorithms, 1 (1990), 51-72.

[6] P. Diaconis and L. Saloffe-Coste, Walks on generating sets of group, Stanford Technical Report No. 481, July 1995, 36 pages.

[7] P. Diaconis and L. Saloffe-Coste, Walks on generating sets of abelian groups, Probab. Theory Relat. Fields, 105 (1996), 393-421.

[8] P. Diaconis and L. Saloffe-Coste, Walks on generating sets of groups, Stanford Technical Report No. 497, July 1996, 40 pages. Probab. Theory Relat. Fields, 105 (1996), 393-421.

[9] P. Diaconis and L. Saloffe-Coste, Logarithmic Sobolev inequalities for finite Markov chains (preprint 1995).

[10] P. Diaconis and M. Shashahani, Time to reach stationarity in the Bernoulli-Laplace diffusion model, SIAM J. Math. Anal., 18 (1987), 208-218. 